\title{
Cardiovascular disease in the Netherlands, 1975 to 1995: decline in mortality, but increasing numbers of patients with chronic conditions
}

\author{
J B Reitsma, J A A Dalstra, G J Bonsel, J H P van der Meulen, R W Koster, \\ L J Gunning-Schepers, J G P Tijssen
}

\begin{abstract}
Objective-To examine the relation between trends over time in mortality and hospital morbidity caused by various cardiovascular diseases in the Netherlands.

Design-Trend analysis by Poisson regression of national data on mortality and hospital admissions from 1975 to 1995. Subjects-The Dutch population.

Results-All cardiovascular diseases combined were responsible for $39 \%$ of all deaths and $16 \%$ of all hospital admissions in 1995. From 1975 to 1995 , age adjusted cardiovascular mortality declined by an annual change of $-2.0 \%(95 \%$ confidence intervals (CI) $-2.1 \%$ to $-1.9 \%$ ), while in the same period age adjusted discharge rates increased annually by $1.3 \%(95 \%$ CI $1.1 \%$ to $1.5 \%$ ). Around $60 \%$ of the gain in life expectancy in this period was related to lower cardiovascular mortality. For mortality, major reductions were seen in coronary heart disease (annual change $-2.9 \%)$ and in stroke $(-2.1 \%)$, whereas the increase in hospital admissions was mainly caused by chronic manifestations of coronary heart disease $(5.1 \%)$, heart failure $(2.1 \%)$, and diseases of the arteries $(1.8 \%)$. In recent years, the gap between men and women at risk of dying from coronary heart disease became smaller for those aged $\leqslant 65$ years.

Conclusions-Our findings of a decrease in cardiovascular mortality and an increase in admission rates for chronic conditions such as heart failure, chronic coronary syndromes, and diseases of the arteries, support the hypothesis that the longer survival of many patients with heart diseases is leading to a growing pool of patients at increased risk for subsequent cardiovascular complications in Western countries. (Heart 1999;82:52-56)
\end{abstract}

Keywords: epidemiology; time trends; mortality; hospital admissions; Netherlands

Cardiovascular diseases (CVD), including stroke and diseases of the arteries, are the leading cause of death in many countries. ${ }^{1-3}$ Moreover, CVD in Western countries are responsible for one fifth of all disability adjusted life years, a measure that combines both years of life lost and years lived in disabil- ity rather than in full health. ${ }^{4}$ In a recent report on the cost of diseases in the Netherlands, CVD accounted for about $11 \%$ of all health care costs in 1994, ranking second after mental disorders. $^{5}$

There has been a remarkable decline in mortality from coronary heart disease over the past 20 years, a result of a decline in incidence as well as of a longer survival among patients with coronary heart disease. ${ }^{67}$ Some researchers have hypothesised that the longer survival of patients with coronary heart disease will result in a growing pool of patients at high risk from subsequent vascular events, and will lead to a higher prevalence of chronic heart conditions. $^{8-11}$

In this report we assess the present burden of CVD in terms of the number of hospital admissions and deaths caused by these diseases. We also seek circumstantial evidence for the above mentioned hypothesis by analysing trends in mortality and morbidity caused by several CVD in the Netherlands simultaneously. Trends were analysed according to age, sex, and calendar year. Special attention was given to coronary heart disease, stroke, diseases of the arteries, and heart failure.

\section{Methods}

Data on the number of deaths in the Netherlands from 1975 to 1995 inclusive were obtained from Statistics Netherlands in Voorburg. Mortality data were grouped by five year age categories, sex, and primary cause of death. The eighth version of the International Classification of Diseases (ICD) was used for the years 1975 to 1978 and the ninth version thereafter. The total group of CVD consisted of all codes from group VII of the ICD classification (diseases of the circulatory system), together with ICD codes identifying congenital anomalies of the circulatory system (in ICD-8 codes 746 and 747; in ICD-9 codes 745-747). The following diseases were analysed separately: coronary heart disease (a combination of ICD codes 410-414), stroke (codes 430-438), and diseases of the arteries (codes 440-448).

The number of hospital admissions for CVD was derived from the central database of hospital admissions in the Netherlands called the National Medical Register. This database is maintained by SIG Health Care Information. In $1975,83 \%$ of all hospital admissions in the Netherlands was recorded in this database. Cover had grown to $95 \%$ in 1980 , and was complete from 1986 onwards. These percent- 
Table 1 Some major causes of death in the Netherlands (1995)

\begin{tabular}{lccc}
\hline Cause of death & Men & Women & Men and women \\
\hline Diseases of the circulatory system & $25635(38 \%)$ & $26694(40 \%)$ & $52329(39 \%)$ \\
Cancer & $20435(30 \%)$ & $16054(24 \%)$ & $36489(27 \%)$ \\
Diseases of the respiratory system & $6970(10 \%)$ & $5674(8 \%)$ & $12644(9 \%)$ \\
Diseases of the digestive system & $2101(3 \%)$ & $2943(4 \%)$ & $5044(4 \%)$ \\
Injury and poisoning & $3033(4 \%)$ & $2140(3 \%)$ & $5173(4 \%)$ \\
Other & $10062(15 \%)$ & $13934(21 \%)$ & $23996(18 \%)$ \\
All cause mortality & 68236 & 67439 & 135675 \\
\hline
\end{tabular}

Source: Statistics Netherlands.

${ }^{\star}$ Including coronary heart disease, stroke, diseases of the arteries, and congenital heart disease.

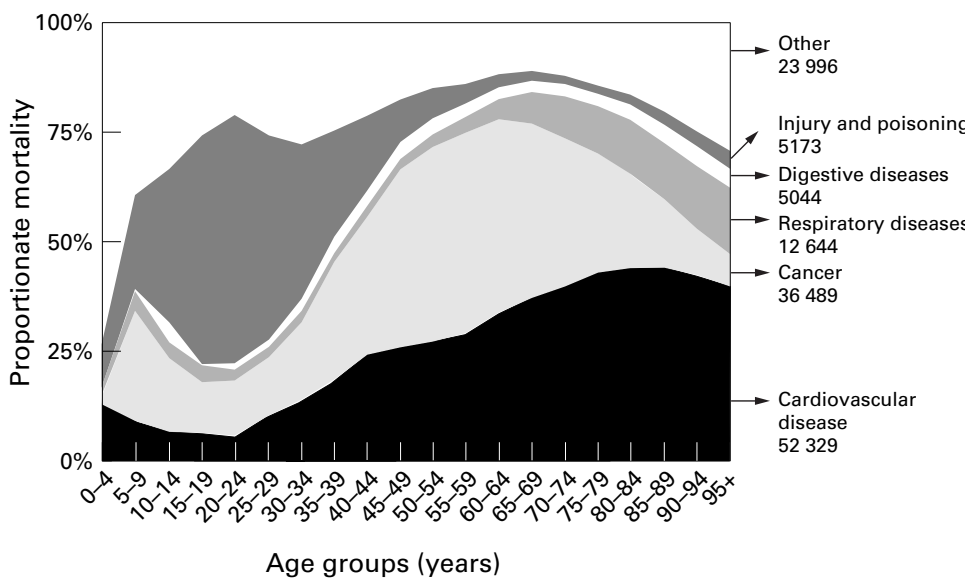

Figure 1 Proportionate mortality by age in the Netherlands in 1995. Men and women combined. Source: Statistics Netherlands.

ages were used to estimate the yearly number of hospital admissions for CVD in the Netherlands. Records contain diagnosis at discharge, age and sex of the patient, length of stay, and status at discharge (dead or alive). Multiple records from the same individual (readmissions) cannot be identified. All diagnoses at discharge were coded according to the Clinical Modification of the International Classification of Diseases (ICD-CM). The switch from the eighth to the ninth version occurred in 1980 . Only admissions with a first listed (primary) discharge diagnosis of one of the CVD were included in this study. Compared with mortality, one more condition was analysed separately: heart failure (ICD-CM-8 codes 427.0, 427.1, and 402; ICD-9-CM codes 428, 402, and 429.1). Furthermore, coronary heart disease was divided into acute coronary syndromes (410 and 411) and chronic coronary syndromes $(412,413$, and 414$)$.

In-hospital case fatality was defined as the proportion of admissions with a discharge status of dead to the total number of admissions for a specific disease. Age adjusted discharge and mortality rates were calculated by direct standardisation to the European Standard Population using equal weighting schemes for men and women. ${ }^{12}$ Life expectancies and lifetime probabilities of dying from CVD were calculated using abridged, current life table techniques. For the conversion of age specific rates to probabilities of dying we used Chiang's method. ${ }^{13}$ Annual relative changes in mortality or discharge rates were estimated by Poisson regression. ${ }^{14}{ }^{15}$ The number of deaths or discharges were used as response variables using the logarithm (ln) of the population size as the offset variable. ${ }^{15}$ Calendar year (con- tinuous), sex, and five year age groups (categorial) were used as explanatory variables. Pearson's $\chi^{2}$ was used to adjust the standard error of the estimates if overdispersion occurred. ${ }^{15}$ For all Poisson trend analyses the GENMOD procedure (generalised linear models) in SAS for Windows version 6.12 was used.

\section{Results}

ALL CVD COMBINED: PRESENT BURDEN AND TRENDS

The total group of CVD was responsible for the deaths of 52329 men and women in 1995, which is equivalent to $39 \%$ of all deaths in the Netherlands (table 1). All cancers combined caused 36489 deaths in 1995, corresponding to $27 \%$ of all deaths. Proportionate mortality by age for the main causes of death is shown in fig 1 . Figure 1 can be divided into four main segments, each linked with a predominant cause of death: from birth to 5 years, perinatal conditions and congenital diseases; from 5 to 34 years, injuries and suicide; from 35 to 64 years, cancer and to a lesser degree CVD; and 65 years and older, CVD and to a lesser degree cancer.

Age adjusted mortality from all CVD in the Netherlands decreased steadily from 419 per 100000 in 1975 to 283 per 100000 in 1995. In the same period, all cause mortality dropped from 934 per 100000 to 760 per 100000 . As a result, life expectancy at birth for men in this period rose by 3.1 years to 74.6 years in 1995 , and for women by 2.7 years to 80.5 years. Fifty four per cent of the gain in life expectancy for men and $63 \%$ for women could be explained by the lower mortality from CVD.

In contrast to the decline in mortality with an annual change of $-2.0 \%$ (95\% confidence interval (CI) $-2.1 \%$ to $-1.9 \%$ ), there was a constant increase in age adjusted discharge rates for CVD with an annual growth of $1.3 \%$ (95\% CI $1.1 \%$ to $1.5 \%$ ). The absolute number of hospital admissions for CVD nearly doubled from 142522 in 1975 (10\% of all hospital admissions in the Netherlands) to 248308 in 1995 ( $16 \%$ of all hospital admissions).

\section{TIME TRENDS FOR DIFFERENT CVD}

Figure 2 combines the trends in age adjusted mortality and discharge rates for several CVD. For mortality, major reductions were seen for coronary heart disease (annual change of $-2.9 \% ; 95 \%$ CI $-3.0 \%$ to $-2.8 \%$ ) and stroke $(-2.1 \% ; 95 \%$ CI $-2.3 \%$ to $-2.0 \%)$, whereas mortality from arterial diseases remained more or less stable $(0.3 \%$; $95 \%$ CI $0.0 \%$ to $0.6 \%)$. Although the relative decline in mortality from coronary heart disease and stroke over the whole study period was similar in magnitude, trends over time were different. During the first half of the study period (1975 to 1985), the rate of decline was faster for stroke (annual change of $-3.0 \%$; $95 \%$ CI $-3.3 \%$ to $-2.8 \%$ ) than for coronary heart disease $(-2.3 \% ; 95 \% \mathrm{CI}-2.4 \%$ to $-2.1 \%)$. During the second part of the study period, coronary heart disease mortality continued to decline at an even higher rate of $-3.7 \%$ (95\% CI $-3.9 \%$ to $-3.4 \%$ ), whereas 

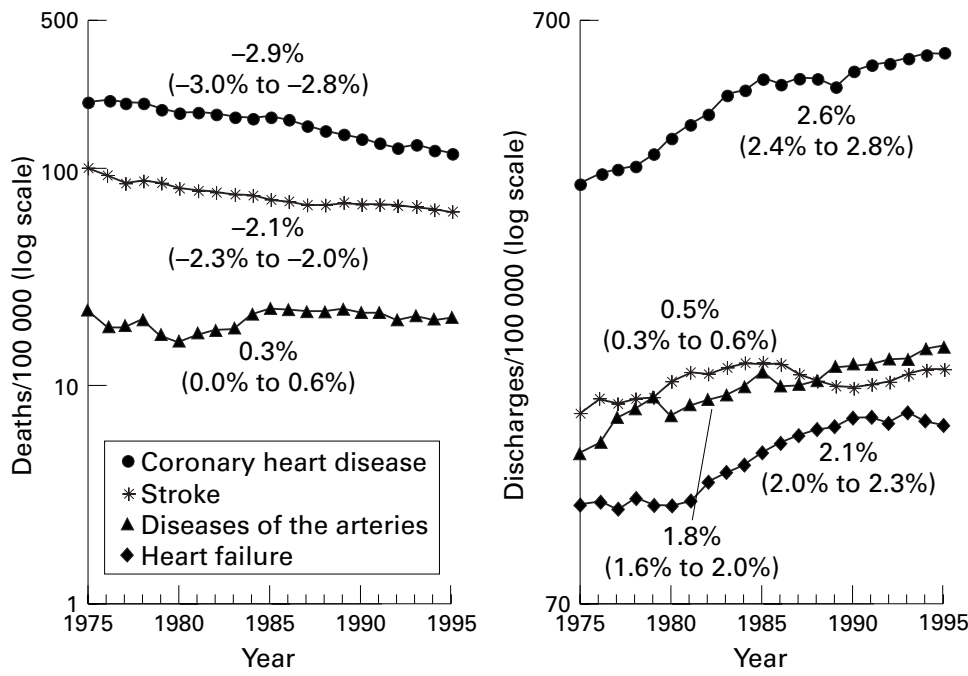

Figure 2 (Left) Trends in age adjusted mortality and (right) discharges rates for several CVD from 1975 to 1995 in the Netherlands. Men and women combined. Direct standardisation to the European Standard Population. ${ }^{12}$ The annual relative change (95\% CI), as estimated by Poisson regression, is presented in the graph. Source: SIG Health Care Information and Statistics Netherlands.

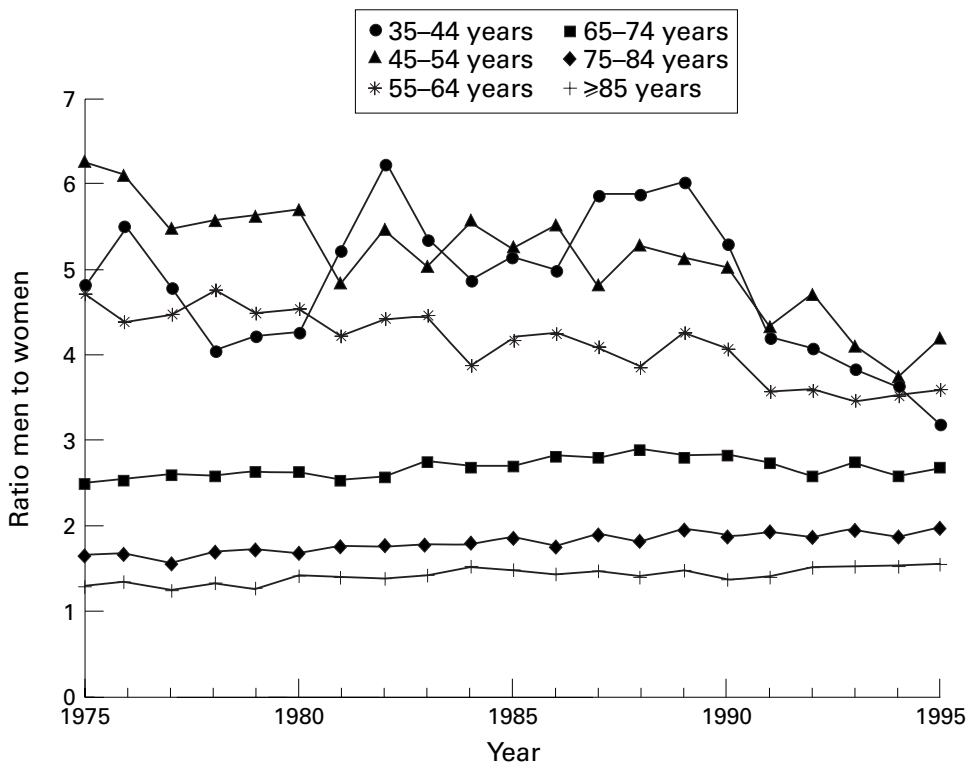

Figure 3 Age specific sex ratios for coronary heart disease mortality from 1975 to 1995. Source: Statistics Netherlands.

mortality from stroke slowed down with an annual relative change of $-1.0 \%(95 \%$ CI $-1.3 \%$ to $-0.7 \%$ ).

For hospital admissions, striking increases were seen in admissions for coronary heart disease (annual growth of $2.6 \% ; 95 \%$ CI $2.4 \%$ to $2.8 \%$ ) with a remarkable difference between acute syndromes ( $0.7 \%$ growth) and chronic manifestations (5.1\% annual growth), for heart failure $(2.1 \%$ annual growth; $95 \%$ CI $2.0 \%$ to $2.3 \%$ ), and for arterial diseases $(1.8 \%$ annual growth; $95 \%$ CI $1.6 \%$ to $2.0 \%$ ). Age adjusted hospital case fatality improved for all major CVD: hospital case fatality for coronary heart disease declined from $12.7 \%$ in 1975 to $4.2 \%$ in 1995 , for stroke from $28.6 \%$ to $17.0 \%$, and for arterial diseases from $9.4 \%$ to $5.4 \%$.
CVD: DIFFERENCES BY SEX AND AGE

If we subject a hypothetical cohort of 100000 men and 100000 women to the age specific mortality risks of CVD as measured in 1995, eventually 38569 men (lifetime probability of 0.39 ) and 40761 women (lifetime probability of 0.41) will die from CVD. Although the lifetime probability of dying from CVD is higher in women than in men, the age at which they die is very different. Of all 40761 women that would die from CVD, the mean age at death would be 82.3 years, with $82 \%$ of these women dying after age 75 . For men the mean age at death would be 76.4 years, with $61 \%$ dying after age 75 .

The rate of decline in mortality from CVD varied among different age groups. In general, the rate of decline was faster in younger age groups. For instance, the relative decline in CVD mortality for those younger than 75 years was $-2.4 \%(95 \%$ CI $-2.5 \%$ to $-2.3 \%)$ compared with $-1.8 \%$ (95\% CI $-1.9 \%$ to $-1.6 \%)$ for those 75 years and older. The annual increase in discharge rates was in general more pronounced in older age groups. The annual growth in the age groups above 75 years was $2.0 \%$ (95\% CI $1.9 \%$ to $2.1 \%$ ) compared with $1.1 \%$ annual growth $(95 \%$ CI $0.9 \%$ to $1.4 \%$ ) in the age groups younger than 75 years.

Generally, trends in mortality from different CVD showed similar patterns among men and women. Only for mortality from diseases of the arteries were trends in the opposite direction observed, with men having an annual increase of $1.3 \%$ (95\% CI $1.0 \%$ to $1.6 \%)$ and women an annual decline of $-1.0 \%(95 \% \mathrm{CI}-1.3 \%$ to $-0.6 \%)$. In recent years, different trends by sex and age were observed for coronary heart disease mortality. Figure 3 presents the age specific sex ratios for coronary heart disease mortality. The following observations can be made. Firstly, in all age groups sex ratios were above 1 , indicating that men had a higher rate of mortality than women. Secondly, sex ratios became smaller with increasing age. Thirdly, sex differences in the age groups below 65 years of age were severely reduced in recent years, whereas sex differences in the older age groups remained more or less stable.

\section{Discussion}

Our analysis showed that CVD are still the leading cause of death in the Netherlands, despite a $30 \%$ decline in age adjusted mortality during the past 20 years. Based on the death rates observed in 1995, the lifetime probability of dying from CVD is $40 \%$ for both men and women, although the mean age at which they die is higher in women than in men. The combined analysis of trends in mortality and morbidity caused by different CVD in the Netherlands revealed an ongoing decline in mortality from CVD, but a continuous increase in the number of hospital admissions for these diseases. It has been hypothesised that the link between the decrease in mortality and the increase in admission rates is the longer survival of patients with CVD, particularly those patients who have had an acute myocardial infarction. ${ }^{8-11}$ The longer survival of 
heart patients will lead to a growing pool of individuals at high risk for subsequent events or diseases, such as recurrent coronary artery stenosis, stroke or congestive heart failure. Before looking at our results in more detail to see if they support this hypothesis, we will first review the literature for the evidence of a longer survival of patients with coronary heart disease.

DECLINE IN MORTALITY FROM CORONARY HEART DISEASE: LOWER INCIDENCE AND LONGER SURVIVAL

Several studies have tried to determine how much of the decline in mortality from coronary heart disease has been caused by a lower incidence of coronary heart disease (primary prevention) and how much by a longer survival (secondary prevention, improvements in medical care).$^{6-9}{ }^{16-20}$ Estimates of the importance of a lower incidence varied between $30 \%$ and $80 \%$ for the different studies analysing the decline in mortality in the 1970s and '80s. ${ }^{6}{ }^{76-20}$ Important lifestyle factors that have led to a lower incidence are a reduction in the number of people who smoke, the treatment of hypertension, and a reduction in the prevalence of hypercholesterolaemia. Studies focusing on the more recent decline in coronary heart disease point to a longer survival among patients with established (coronary) heart disease as an important factor in the continuing decline in mortality. ${ }^{89}$ The following factors were specifically mentioned: coronary bypass surgery, coronary angioplasty, thrombolysis in acute coronary syndromes, and secondary medical prevention in patients with myocardial infarction (antiplatelet agents, anticoagulants, and $\beta$ blockers) to prevent vascular events.

Several findings from our study also indicate a longer survival among patients with coronary heart disease, especially in recent years. Firstly, there was a substantial decline in hospital case fatality for many CVD, especially for coronary heart disease. Secondly, time trends for recent years (from the mid-1980s) were different for various CVD. The annual relative decline in mortality from coronary heart disease increased even further, whereas the decline in mortality from stroke and diseases of the arteries slowed down.

LONGER SURVIVAL BUT MORE PATIENTS WITH CHRONIC CONDITIONS

It has been hypothesised that the longer survival of patients with coronary heart disease will lead to a growing group of patients at high risk from subsequent vascular events, causing an increase in the prevalence of chronic conditions. ${ }^{8-11}$ The remarkable growth in the number of hospital admissions for CVD and the type of diseases responsible for this increase are in line with this hypothesis. Major increases were seen for congestive heart failure, chronic coronary syndromes, and arterial diseases (fig $2)$. In the interpretation of the increase in hospital admissions it is important to recognise that multiple admissions of the same patient cannot be determined. In the case of chronic conditions like heart failure and arterial diseases, readmissions might be frequent. What proportion of the increase in hospital admissions is related to readmissions and how much to "new" patients remains unclear. We have already demonstrated that in heart failure patients multiple readmissions within a short period of time are common. ${ }^{21}$ An additional factor in the rise of the number of admissions for coronary heart disease is the intensive use of diagnostic and therapeutic procedures in patients with coronary syndromes.

DIFFERENCES IN TIME TRENDS FOR MORTALITY FROM CORONARY HEART DISEASE BETWEEN MEN AND WOMEN

A significant finding of this study is that the gap between men and women at risk of dying from coronary heart disease has become smaller in recent years for those aged 65 years and younger (fig 3). The reasons for this are not clear, but might be related to men having more benefit from recent advances in medical care or women adopting more unfavourable lifestyles. Several studies have demonstrated that women with coronary heart disease are treated differently from men. ${ }^{22-25}$ These differences include a lower use of invasive diagnostic testing in women, lower rates of revascularisation in women, and less likelihood of women being discharged with aspirin and $\beta$ blocking agents. The fact that women with myocardial infarction are in general older and have more traditional risk factors such as hypertension, diabetes mellitus, and congestive heart failure than men at the time of admission, could not fully explain the lower use of thrombolysis in women. ${ }^{26}$

STRENGTHS AND LIMITATIONS OF THE STUDY This was a descriptive study, analysing only temporal relations in data from two national registries. The validity of this study depends strongly on the accuracy of the primary cause of death or the primary diagnosis at discharge. For mortality, discrepancies have been found between the judgment of physicians and subsequent findings at necropsy, and between physicians coding identical cases for research purposes. ${ }^{27-30}$ The use of broad categories of diseases, as has been done in this study, is known to lead to fewer discrepancies than analysing single disorders. ${ }^{29} 30$

The use of hospital statistics data is limited by the inability to identify multiple admissions of the same patient. Furthermore, the number of hospital admissions is affected by changes in admission policy and by improvements in diagnostic capabilities. The significance of these factors will vary with the disease under study. They will be of minor importance for diseases like acute myocardial infarction, in which nearly all patients are hospitalised, but become more important for diseases like stroke (introduction of computed tomography) and heart failure (coding problems and admission policy). The lower hospital case fatality could have been influenced by more admissions for milder forms, by more frequent readmissions, and by more admissions for diagnostic purposes. 
This study underlines the dynamic and complex interactions that exist between morbidity and mortality caused by different CVD. Studies with a limited time of follow up or trials dealing with selected patients will reveal only part of the total picture important from a public health point of view. More attention needs to be given to the exact benefits, both short and long term, of new additions to the treatment of heart patients and to differences in medical care and outcomes between men and women. The shift from acute and fatal to more chronic conditions should lead to more attention being focused on the reasons for and prevention of readmissions in patients with chronic disorders. $^{31}$

To summarise, CVD are still the leading cause of death among men and women in the Netherlands, despite a major decline in age adjusted mortality of more than $30 \%$ in both men and women. This decline was the major reason behind the gain in life expectancy. In the same period that mortality declined, the number of hospital admissions for CVD rose steadily. This increase was mainly caused by diseases related to non-acute syndromes of coronary heart disease, heart failure, and arterial diseases. These findings support the hypothesis that improvements in medical care have resulted in a longer survival of many heart disease patients, and led to a growing prevalence of patients with chronic cardiovascular conditions. These growing numbers of patients, coupled with the increased aging of many European populations, will increase the strain on forthcoming health care budgets even further. ${ }^{32}$ The reduced difference in mortality from coronary heart disease between men and women aged 65 years and younger might suggest that men have had more benefit from the recent improvements in medical care than women.

This work was supported by a grant from the Netherlands Heart Foundation (grant number 42.012). For this project an advisory committee was installed by the Netherlands Heart Foundation. We are indebted to the members of this committee for their helpful comments during the preparation of the paper.

1 Thom TJ, Epstein FH. Heart disease, cancer, and stroke mortality trends and their interrelations. An international perspective. Circulation 1994;90:574-82.

2 Thom TJ. International mortality from heart disease: rates and trends. Int $\mathcal{F}$ Epidemiol 1989;18(suppl 1):S20-8.

3 Uemura K, Pisa Z. Trends in cardiovascular disease mortality in industrialised countries since 1950. World Health Stat $Q 1988 ; 42: 155-77$

4 Murray CJL, Lopez AD, eds. The global burden of disease. Volume I: A comprehensive assessment of mortality and disability from diseases, injuries, and risk factors in 1990 and projected to fom Organisation, World Bank, 1996.

5 Polder JJ, Meerding WJ, Koopmanschap MA, et al. Costs of diseases in the Netherlands 1994 [in Dutch]. Report of the Department of Public Health and Social Medicine and the Department of Public Health and Social Medicine and the Erasmus University, 1997.
6 Goldman L, Cook EF. The decline in ischemic heart disease mortality rates: an analysis of the comparative effects of medical interventions an

7 Sytkowski PA, Kannel WB, D'Agostino RB. Changes in risk factors and the decline in mortality from cardiovascular disease: the Framingham heart study. N Engl F Med 1990; 322:1635-41.

8 McGovern PG, Pankow JS, Shahar E, et al. Recent trends in acute coronary heart disease: mortality, morbidity, medical care, and risk factors. $N$ Engl $₹$ Med 1996;334:884-90.

9 Hunink MG, Goldman L, Tosteson ANA, et al. The recent decline in mortality from coronary heart disease, 1980990: the effect of secular trends in risk factors and treatment. $\mathcal{F} A M A$ 1997;277:535-42.

10 Bonneux L, Looman CWN, Barendregt JJ, et al. Regression analysis of recent changes in cardiovascular morbidity and mortality in the Netherlands. BMF 1997;314:789-92.

11 Stevenson R, Ranjadayalan K, Wilkinson P, et al. Short and long term prognosis of acute myocardial infarction since introduction of thrombolysis. BMF 1993;307:349-53.

12 World Health Organisation. World Health Statistics Annual 1995. Geneva: WHO, 1996:XXV.

13 Chiang CL. On constructing current life tables. I Am Stat Assoc 1972;67:538-41.

14 Breslow NE, Day NE. Fitting models to grouped data. In: Heseltine E, ed. Statistical methods in cancer research. Volume II: the design and analysis of cohort studies. New York: Oxford University Press, 1987.

15 McCullagh P, Nelder JA. Log-linear models. Generalized linear models. London: Chapman and Hall, 1989:127-48.

16 Bots ML, Grobbee DE. Decline of coronary heart disease mortality in the Netherlands from 1978 to 1985: contribution of medical care and changes over time in presence of major cardiovascular risk factors. F Cardiovasc Risk 1996;3: 271-6.

17 Goldman L, Cook F, Hashimoto B, et al. Evidence that hospital care for acute myocardial infarction has not contributed to the decline in coronary mortality between 1973-1974 and 1978-1979. Circulation 1982;65:936-42.

18 Jamrozik K, Hockey R. Trends in risk factors for vascular disease in Australia. Med f Aust 1989;150:14-8.

19 Sigfusson N, Sigvaldason H, Steingrimsdottir L, et al. Decline in ischaemic heart disease in Iceland and change in risk factor levels. BMf 1991;302:1371-5.

20 Vartiainen E, Puska P, Pekkanen J, et al. Changes in risk factors explain changes in mortality from ischaemic heart disease in Finland. BMF 1994;309:23-7.

21 Reitsma JB, Mosterd A, de Craen AJM, et al. Increase in hospital admission rates for heart failure in the Netherlands, 1980-1993. Heart 1996;76:388-92.

22 Ayanian JZ, Epstein AM. Differences in the use of procedures between women and men hospitalized for coronary heart disease. N Engl f Med 1991;325:221-5.

23 Shaw LJ, Miller DD, Romeis JC, et al. Gender differences in the noninvasive evaluation and management of patients with suspected coronary artery disease. Ann Intern Med 1994;120:559-66.

24 Jaglal SB, Goel V, Naylor CD. Sex differences in the use of invasive coronary procedures in Ontario. Can $\mathcal{f}$ Cardiol 1994;10:239-44.

25 Clarke KW, Gray D, Keating NA, et al. Do women with acute myocardial infarction receive the same treatment as men? BMF 1994;309:563-6.

26 European Secondary Prevention Study Group. Translation of clinical trials into practice: a European population-based study of the use of trombolysis for acute myocardial infarction. Lancet 1996;347:1203-7.

27 Engel LW, Strauchen JA, Chiazze L Jr, et al. Accuracy of death certification in an autopsied population with specific attention to malignant neoplasms and vascular diseases. Am $\mathcal{F}$ Epidemiol 1980;111:99-112.

28 Mackenbach JP, van Duyne WMJ, Kelson MC. Certification and coding of two underlying causes of death in the tion and coding of two underlying causes of death in the nity. I Epidemiol Comm Health 1987;41:156-60.

29 Messite J, Stellman SD. Accuracy of death certificate completion: the need for formalized physician training. ҰAMA 1996;275:794-6.

30 Kircher T, Nelson J, Burdo H. The autopsy as a measure of accuracy of the death certificate. $N$ Engl F Med 1985;313: 1263-9.

31 Rich MW, Beckham V, Wittenberg C, et al. A multidisciplinary intervention to prevent the readmission of elderly patients with congestive heart failure. N Engl f Med 1995; 333:1190-5.

32 Dall JLC. The greying of Europe. BMF 1994;309:1282-5. 